\title{
A STIMULUS INDEPENDENT GENERATOR FUNCTION FOCUSED UPON MERKEL CELL AND NEURITE CURRENTS OF THE SLOWLY ADAPTING TYPE I CUTANEOUS AFFERENT
}

\author{
A Thesis \\ Presented to \\ the faculty of the School of Engineering and Applied Science \\ University of Virginia \\ in partial fulfillment \\ of the requirements for the degree \\ Master of Science \\ by \\ Lingtian Wan
}

August

2015 
APPROVAL SHEET

The thesis

is submitted in partial fulfillment of the requirements

for the degree of

Master of Science

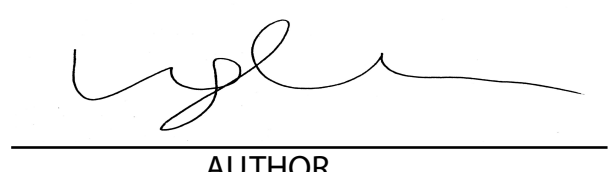

AUTHOR

The thesis has been read and approved by the examining committee:

Gregory Gerling

Advisor

Stephen Patek

Jeff Saucerman

Ellen Lumpkin

Accepted for the School of Engineering and Applied Science:

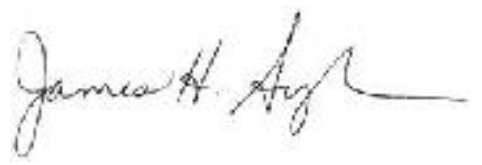

Dean, School of Engineering and Applied Science

August

2015 


\section{Table of Contents}

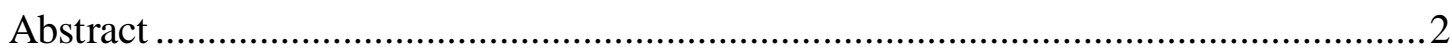

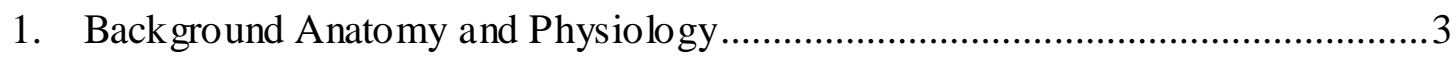

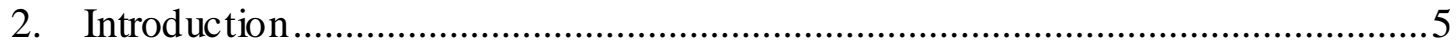

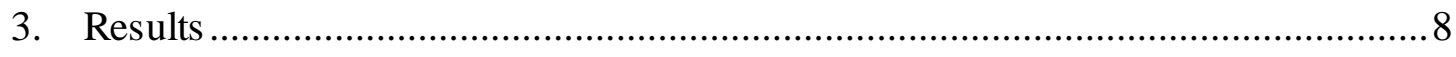

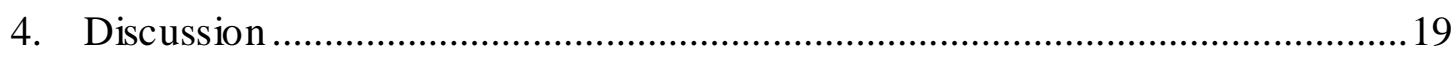

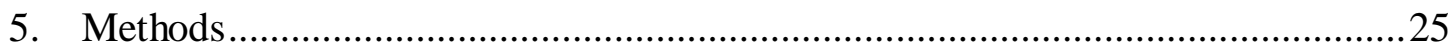

5.1 Conceptual Understanding of the Generator Function .................................. 25

5.2 Mathematical Form of the Generator Function ........................................... 27

5.3 Generator Function in context of SAI Whole End Organ Model................... 28

5.4 Fitting the Parameters of the Generator Function ........................................ 31

5.5 Numerical Experiments with the Generator Function in Context of the End

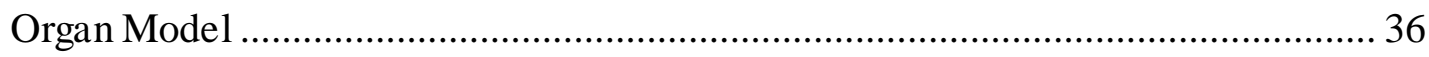

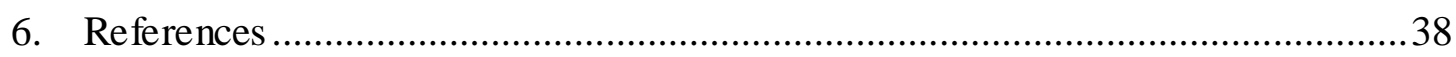




\begin{abstract}
The slowly adapting type I (SAI) cutaneous afferent innervates Merkel cells and their associated neurites, which constitute its architecture as a branching end organ. It is presently unknown how Merkel cells and neurites contribute, respectively, with receptor currents on different timescales, to the slow adaptation observed in elicited trains of action potentials. Due to the inherent difficulty of direct measurement, this effort in computational modeling is inspired by recent findings for Piezo 2 mechanically activated channels in mice. The developed generator function includes components of a Merkel cell mechanism (slower decay of current) and a neurite mechanism (faster decay of current). Each of these functions takes as its input instantaneous stress in the skin, which each linearly convolves with its prior accumulation of decaying current over time. Summed together, recent time histories of stimulus magnitude and rate, as receptor current, are carried to the present. When the Merkel cell mechanism is removed in the context of simulating the entire end organ of the SAI afferent, its characteristic response of sustained spike firing over the hold of the stimulus is attenuated, as is observed for Piezo2 deficient animals. Furthermore, while prior models have directly converted the time derivatives of stimulus position into receptor current, this function uses biological mechanisms to make such conversions, and therefore is not fitted to or dependent on a particular stimulus.
\end{abstract}




\section{Background Anatomy and Physiology}

When we touch an object, mechanosensitive afferents in our fingers convert our skin's deformation into ionic signals that travel through peripheral nerves to our central nervous system. It is from a spatial array of afferents that we form our perception of an object's spatial and temporal features. One class of cutaneous light touch neurons, slowly-adapting type I (SAI) afferents, are distinguished by several characteristics. These include a sustained response to held stimuli, greater spike firing to stimulus movement than stimulus hold, variable length intervals between successive action potentials, or "spikes", and ability to respond over a large range of forces (Figure 1). In specific, when pressure is applied to the skin's surface, internal forces propagate through the skin toward the junction of the epidermis and dermis, where are located clusters of Merkel cells. The SAI afferent innervates Merkel cell end organs (Figure 2). An individual Merkel cell is typically associated with an unmyelinated neurite, which connects to a heminode either directly or along a chain of Merkel cells coupled to their neurites. It is believed that the heminode is the point of action potential generation and before that point only receptor currents are encountered, though at present their magnitudes are difficult to observe. There are multiple heminodes within a Merkel cell-neurite end organ. Eventually, all heminodes tie together at an upstream node which passes information to the central nervous system. 


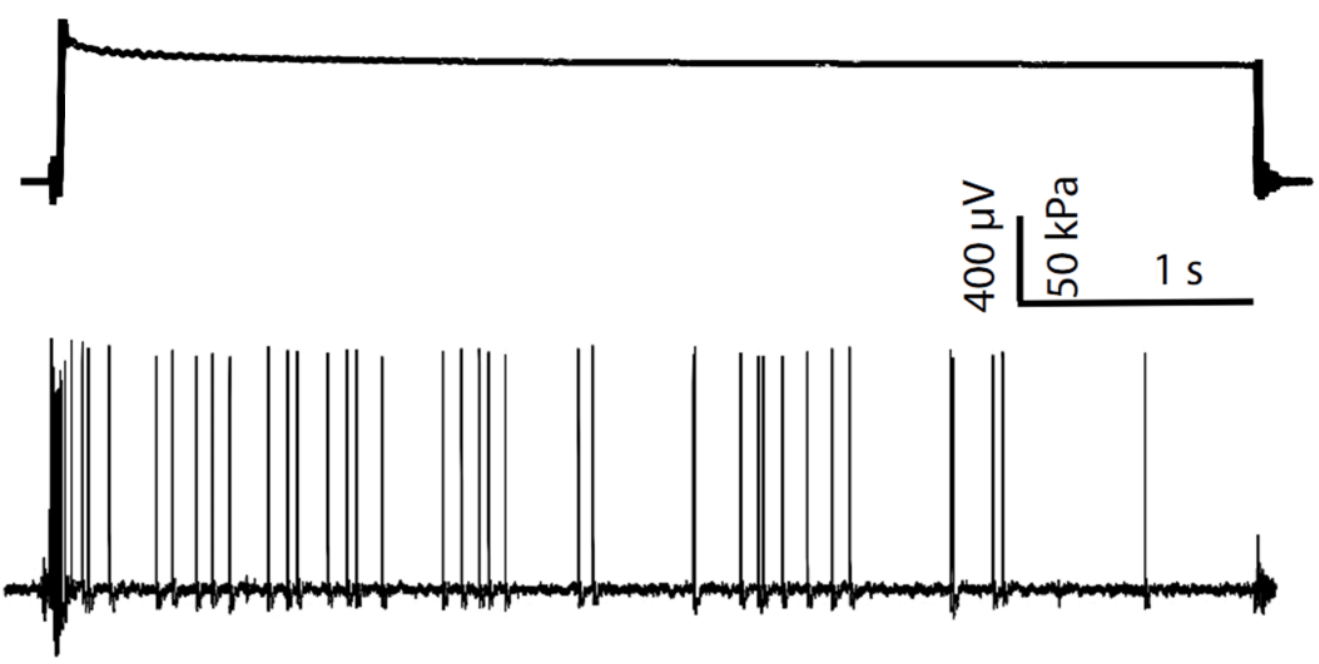

Figure 1. An SAI afferent's response in terms of voltage traces over time (below) showing action potential firing to a ramp-and-hold stimulus of about $80 \mathrm{kPa}$ (above)

(Wellnitz, Lesniak, Gerling, \& Lumpkin, 2010).
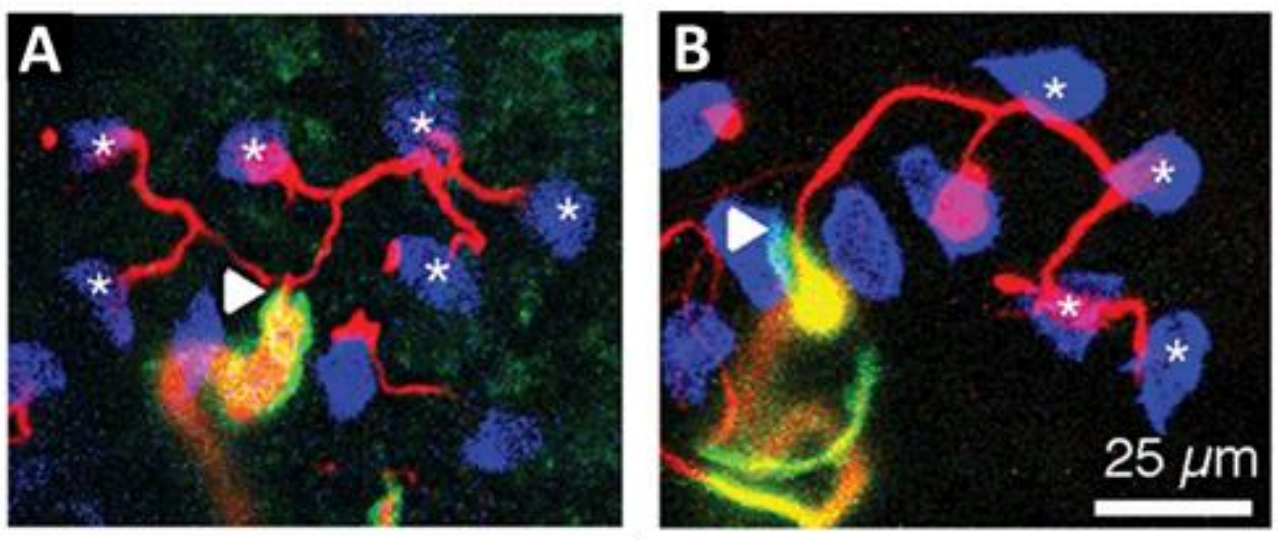
Figure 2. Merkel cells are marked (blue) in proximity to neurites (red) that extend toward a heminode (arrowhead) (Lesniak et al., 2014). In the partial touch dome of case (A), six individual neurites are connected to individual Merkel cells (asterisks) and in case (B) four Merkel cells (asterisks) are connected in in a sequential chain.

\section{Introduction}

The slowly adapting type I (SAI) afferent innervates Merkel cells and their associated neurites, which constitute its architecture as a branching end organ. It is presently unknown how Merkel cells and neurites contribute, respectively, with receptor currents on different timescales, to the slow adaptation observed in elicited trains of action potentials. One means of attaining insight is to consider animals of different genetic backgrounds. For example, those animals deficient in Piezo2 mechanically activated, cation channels in the Merkel cells of the skin, but not in sensory neurons, exhibit a lack of a neural response to the sustained hold of a stimulus, which is a defining characteristic of the SAI afferent (Woo et al., 2014). Another means is to measure currents and potentials at separate junctures in their generation process. For example, under mechanical stimulation, one can presently observe the inward currents for single Merkel cells (Woo et al., 2014), or the rapidly adapting currents in dorsal root ganglion neurons (Ranade et al., 2014). However, it is experimentally difficult to directly measure receptor currents at the level of single neurites, 
and to isolate, respectively, the currents generated by Merkel cell and neurite mechanis ms. The effort herein seeks to further understand these processes by computational modeling how Merkel cells and neurites influence, separately and together, the generation of receptor currents, whose values in the context of an entire end organ give rise to trains of action potentials.

While related models have indeed transformed mechanical stimulation to receptor current, nearly all of their underlying mechanics are directly tied and fitted to the stimulus (Dong et al., 2013; Freeman \& Johnson, 1982b; Kim, Sripati, \& Bensmaia, 2010; Lesniak \& Gerling, 2009; Looft \& Baltensperger, 1990; Sripati, Bensmaia, \& Johnson, 2006), rather than the biophysical interactions of the Merkel cell and neurite (Woo, Lumpkin, \& Patapoutian, 2015) that extend to groups of heminodes of the end organ (Lesniak et al., 2014). In specific, most models directly convert the time derivatives of stimulus position into receptor current, which makes them non-physiologically based and heavily dependent upon parameter fitting to particular surface stimuli. For example, in predicting the timing of individual spikes evoked by mechanical vibrations in three types of mechanoreceptive afferent fibers (Kim et al., 2010), stimulus displacement and its derivatives (position, velocity, acceleration, and jerk) were separately filtered using different temporal linear filters and summed with different weights to form current input to a neural dynamics model. Likewise, in a focus on ramp-and-hold stimuli (Lesniak et al., 2014), stresses and strains 
internal to the skin's layers and their change over time are converted into receptor current. While perhaps a stress term represents a static response similar to the Merkel cell mechanism and its first derivative a dynamic response similar to the neurite mechanism, the mapping of such derivatives to either physiological mechanism is rudimentary and not clearly differentiated from what could also be framed as direct ties to stimulus position and movement.

Therefore, this work designs a generator function inspired by recent findings on Piezo 2 mechanically activated channels in mice, including components related to a Merkel cell mechanism (slower decay of current) and a neurite mechanism (faster decay of current). The above functions filter stress internal to the skin over time, in a linear convolution form, thereby carrying recent stress information forward to the present. Each of these functions takes as its input instantaneous stress in the skin, which each linearly convolves with its prior accumulation of decaying current over time. Summed together, recent time histories of stimulus magnitude and rate, as receptor current, are carried to the present. The parameters of the generator function are derived from physiological recordings of membrane potentials for single Merkel cells and of receptor currents just distal to node locations in SAI afferents. Then, by placing the generator function in the context of an end organ model with multiple Merkel cell-neurite complexes and heminode sites of spike initiation (Lesniak et al., 2014), trains of action potentials, or 'spikes,' are predicted. Within 
the end organ model, the generator function bridges a model of skin mechanics that transforms a surface stimulus to stress internal to the skin's layers and a model of neural dynamics that transforms receptor current to spike times. Numerical experiments, both at the level of single Merkel cell and neurite interaction and at the level of an entire end organ, demonstrate the impact of parameter changes and their interactions. One case in particular considers Piezo2 deficient animals, while another extends to the control of the stimulus.

\section{Results}

Numerical experiments with the generator function demonstrate 1) its base case, 2) the effects of varying its parameters, 3) its ability in a whole end organ context to predict trains of spikes as recorded at a single myelinated axon, 4) the muting of the Merkel cell mechanism to produce spike train responses similar to Piezo2 deficient animals, and 5) that the function can operate under ramp-and-hold stimulation controlled both by displacement and force in addition to periodic vibration.

First, the base case of the generator function shows its current output over 2 seconds, in response to a ramp-and-hold displacement (Figure 3). The current generated by the Merkel cell mechanism (Figure 3A) is sustained during the stimulus hold, with a gradual increase at the ramp-up phase (from contact of skin and stimulus to peak value of stimulation) and slow decay afterwards. In contrast, the current generated by the neurite 
mechanism (Figure 3B) reacts more rapidly to changes in stimulation over the stimulus ramp, and is of a larger magnitude, and quickly decays to zero afterwards. Therefore, the current summed between these two mechanisms (Figure 3C) responds fast and larger in magnitude to the ramp-up phase, decays slowly at early-hold phase (from peak value of stimulation to 0.5 seconds afterwards) in response to the stress relaxation of the skin output from the finite element model, and maintains a steady level through late-hold phase (from 2 to 4.5 seconds of the stimulation).

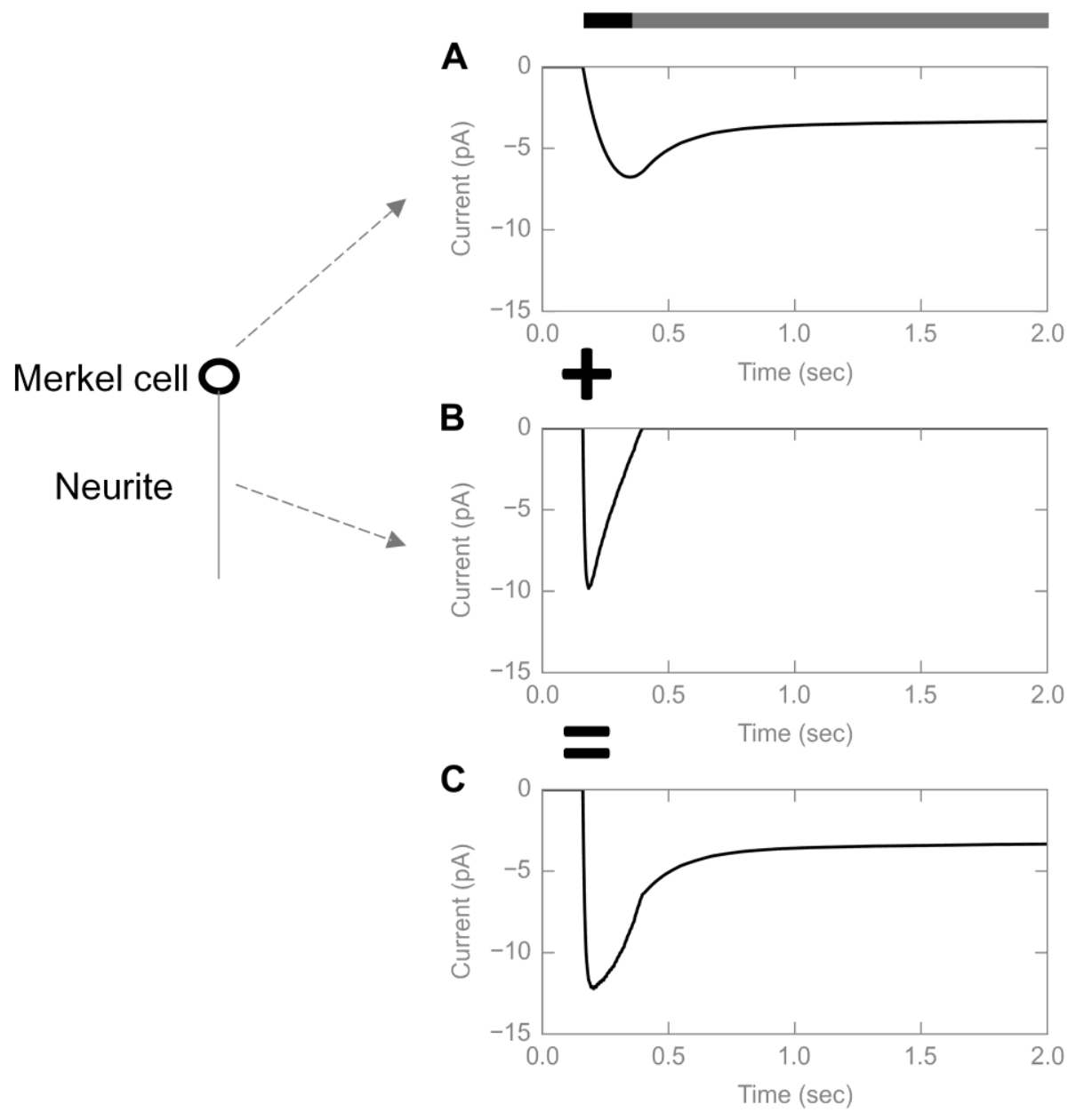


Figure 3. Current output by the generator function over 2 seconds, in response to the ramp-and-hold displacement as shown in the line to the top of the figures with the ramp in the darker portion and the hold in the lighter portion. The three figures show current as generated by (A) the Merkel cell mechanism, (B) the neurite mechanism, and (C) both mechanisms together.

Second, the variation of the generator function's parameters shows its ability to affect the magnitude of current values and eventually the spike firing rate, over different time phases of the response. For example, increasing the decay time constant $\left(\tau_{l}\right)$ of the neurite mechanism increases the current response (Figure 4A) and firing rate (Figure 4B) at the ramp-up phase of stimulation. Increasing the decay time constant $\left(\tau_{2}\right)$ of the Merkel cell mechanism increases the current response (Figure 4C) and firing rate (Figure 4D) of the early-hold phase. Increasing the peak/steady-state magnitude ratio $\left(K_{l}\right)$ of the Merkel cell mechanism increases the current response (Figure 4E) and firing rate (Figure 4F) of the late-hold phase. 

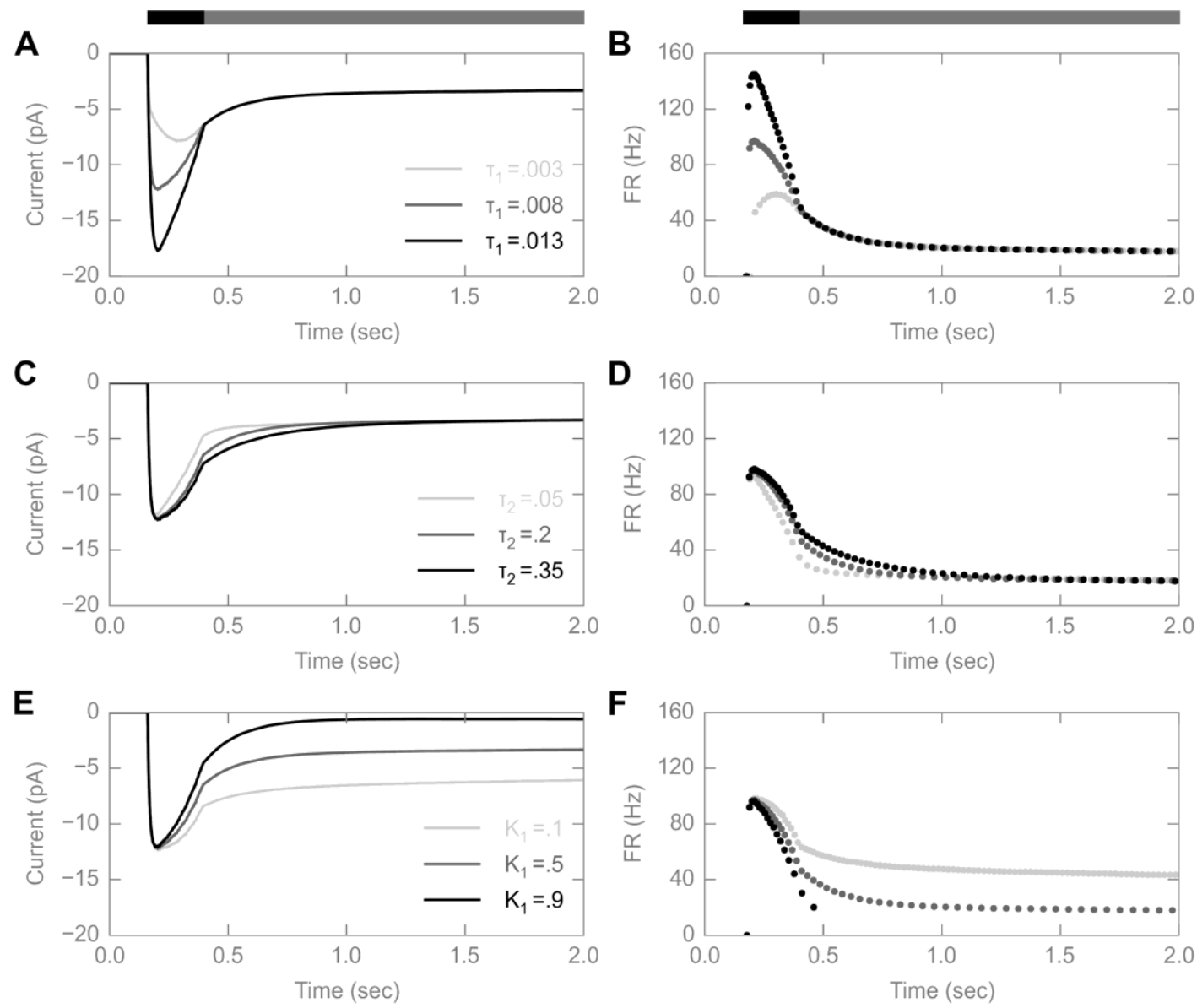

Figure 4. Increasing generator function parameters $\tau_{l}, \tau_{2}$, and $K_{l}$ can increase receptor current in ramp-up, early hold, and late hold phases, respectively, as well as their corresponding firing rates. In particular, current traces with different (A) $\tau_{I}$ values show the impact upon the peak current produced, (C) $\tau_{2}$ values show the impact during the early hold phase, and (E) $K_{l}$ values show the impact upon modulating the steady state magnitude relative to the peak. Figures (B), (D), and (F) show corresponding firing rates when the generator function is run in the context of the entire end organ model. 
Third, when the generator function is used in the context of the entire end organ model, the predicted spike trains (Figure 5A) and firing rates (Figure 5C) over time mimic the recorded spike trains (Figure 5B) and firing rates (Figure 5D), in both magnitude and shape. The firing rates at different phases of the stimulus (ramp-up: early hold: late hold) for both the model predictions and recordings are also similar (Table 1).
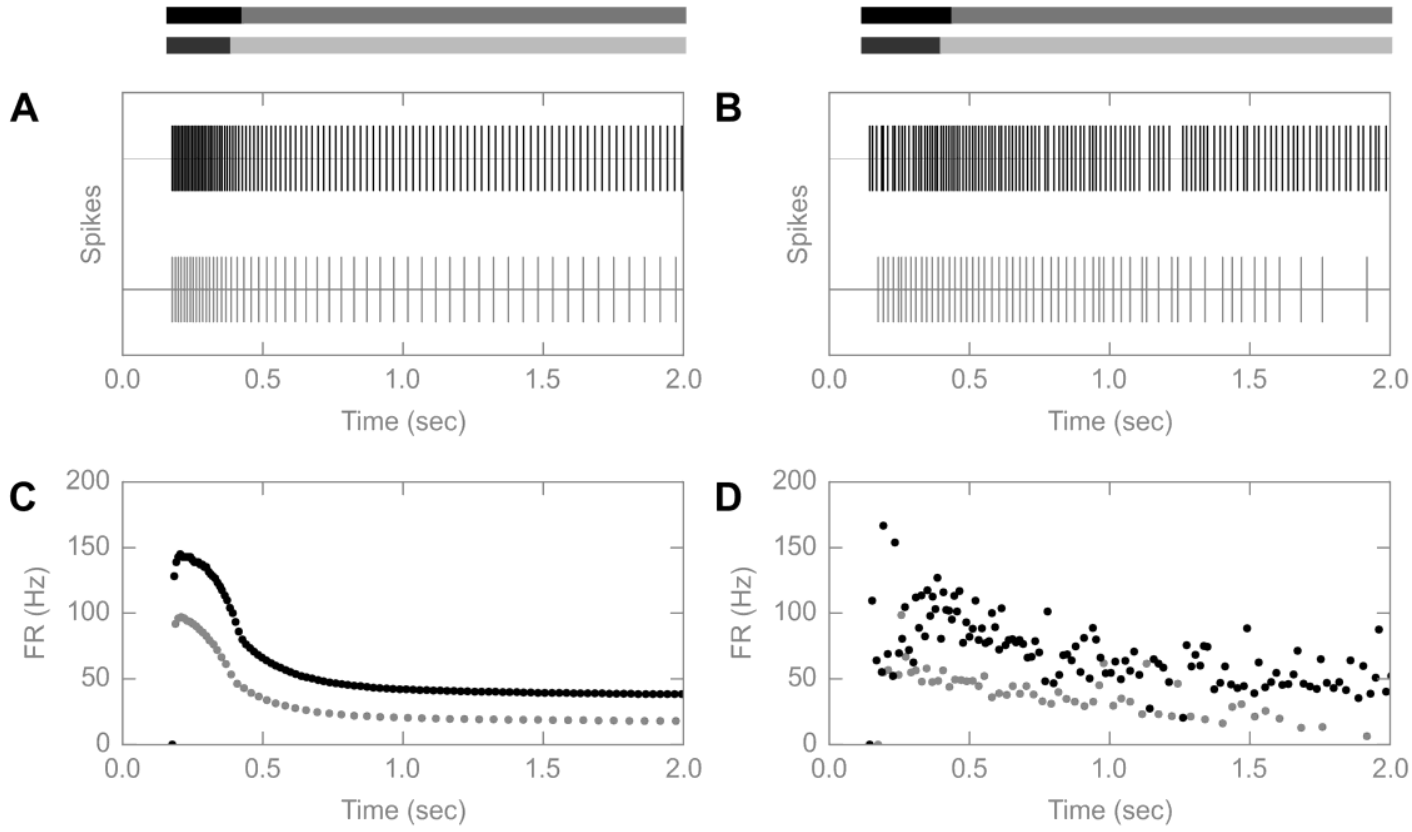

Figure 5. Spike trains and firing rates from both model predictions and

electrophysiological recordings under two ramp-and-hold displacements show similarity in terms of both the magnitude and shape of the firing rate over time. In specific, subfigures (A) and (C) show model predictions, while (B) and (D) show electrophysiological recordings. Note that unlike Lesniak, 2014 there was no noise introduced into this model so there the intervals between spikes are not irregular. 
Table 1. Firing rates (model predictions versus electrophysiological recordings) calculated at three stimulus phases for two displacement levels $(0.45$ and $0.55 \mathrm{~mm})$. The ratio column compares the three phases with each other (ramp-up: stimulation onset to peak; early-hold: peak to $0.5 \mathrm{sec}$ after peak; late-hold: 2 - $4.5 \mathrm{sec}$ after onset).

\begin{tabular}{|c|c|c|c|c|}
\hline $\begin{array}{c}\text { Model predictions vs } \\
\text { Recordings (Hz) }\end{array}$ & Ramp-up & Early-hold & Late-hold & Ratio \\
\hline Displacement 1 & 57 vs 85 & 41 vs 45 & 14 vs 17 & $4.3: 3: 1$ vs 5.1:2.6:1 \\
\hline Displacement 2 & 98 vs 129 & 75 vs 75 & 44 vs 37 & $2.2: 1.7: 1$ vs 3.5:2:1 \\
\hline
\end{tabular}

Fourth, the muting of the Merkel cell mechanism in the generator function predicts neural responses consistent with electrophysiological recordings from those mice deficient in Piezo2 channels in the skin (Figure 6) (Woo et al., 2014). With both mechanisms on, spike firing continues throughout the stimulation, while with only neurite mechanism on, the firing stops long before the end of the stimulation. 

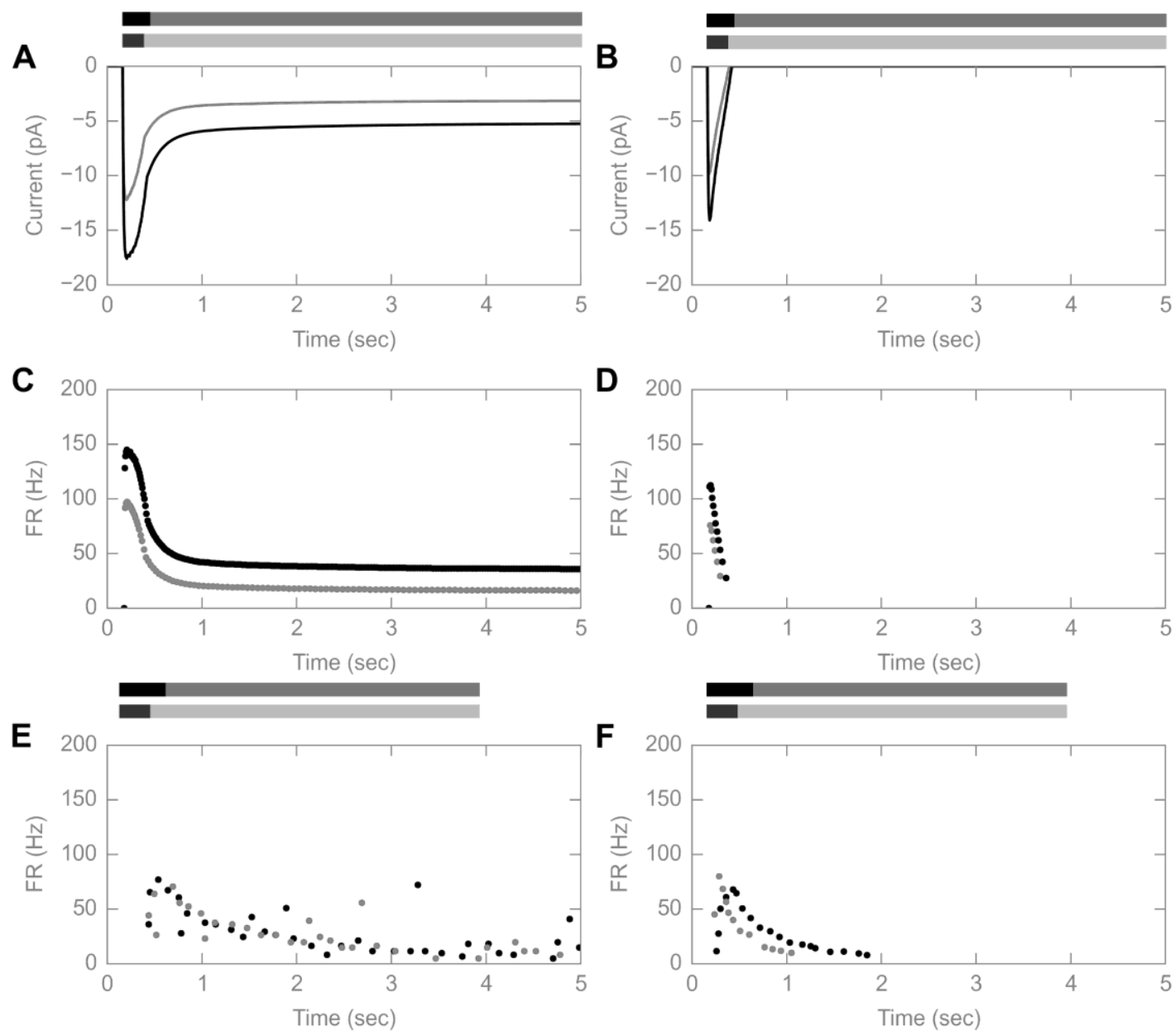

Figure 6. The muting of the Merkel cell mechanism in the generator function predicts neural responses consistent with electrophysiological recordings from those mice deficient in Piezo2 channels in the skin, under two levels of ramp-and-hold displacement. In particular, shown are (A) currents from the complete function and (B) currents with only the neurite mechanism. The results indicate that firing rates from the (C) complete generator function have steady, non-zero values throughout the stimulus hold and (D) function with only neurite mechanism lack a response in the sustained hold. For reference 
are shown firing rates from electrophysiological recordings in (E) Piezo2 control mice with steady, non-zero values throughout the stimulus hold and (F) Piezo2 deficient mice that lack a response in the sustained hold (Woo et al., 2014).

Fifth, the generator function can operate under ramp-and-hold stimulation controlled both by displacement and force in addition to sinusoidal vibration. Force-controlled stimulations (Figure 7A, dashed lines) that generate similar stress maintained over time in the skin were compared with displacement-controlled stimulations (Figure 7A, solid lines and dots) whereby stress decays over time. The generator function captures the difference between the two control strategies by outputting current (and subsequently firing rates) with less difference between peak and steady-state values for force-controlled stimulations (Figures 7B and 7C) that displacement-controlled stimulations. Another way to view this same trend is via a comparison of the ratios of firing rates between phases of the stimulus (ramp to peak: early hold of peak to $0.5 \mathrm{sec}$ : late hold of 2-4.5 sec) for force-controlled (2.6: 1.5: 1) during the entire stimulation vary less than the displacement-controlled firing rates (4.3: 2.3: 1). In addition, stimulation using half sinusoids (only for the portion indented from skin contact deeper into the skin, not from skin contact to stimulus in air) at frequencies of $5 \mathrm{~Hz}$ (Figure 8A), $10 \mathrm{~Hz}$ (Figure 8B) and $20 \mathrm{~Hz}$ (Figure 8C) were compared with recordings from the primate (Freeman \& Johnson, 1982a). For each frequency, the 
predicted increase in impulse rate of 0 to 2 with an increase to vibration amplitude mimics the experimental data. Furthermore, stimulation at a $1 \mathrm{~Hz}$ frequency was compared with the ramp-and-hold stimulation (Figure 9) to show that the two match in terms of spikes/cycle as the frequency of the sinusoid decreases, and to put low frequency stimulation in the context of the rate of the ramp in a ramp-and-hold displacement.
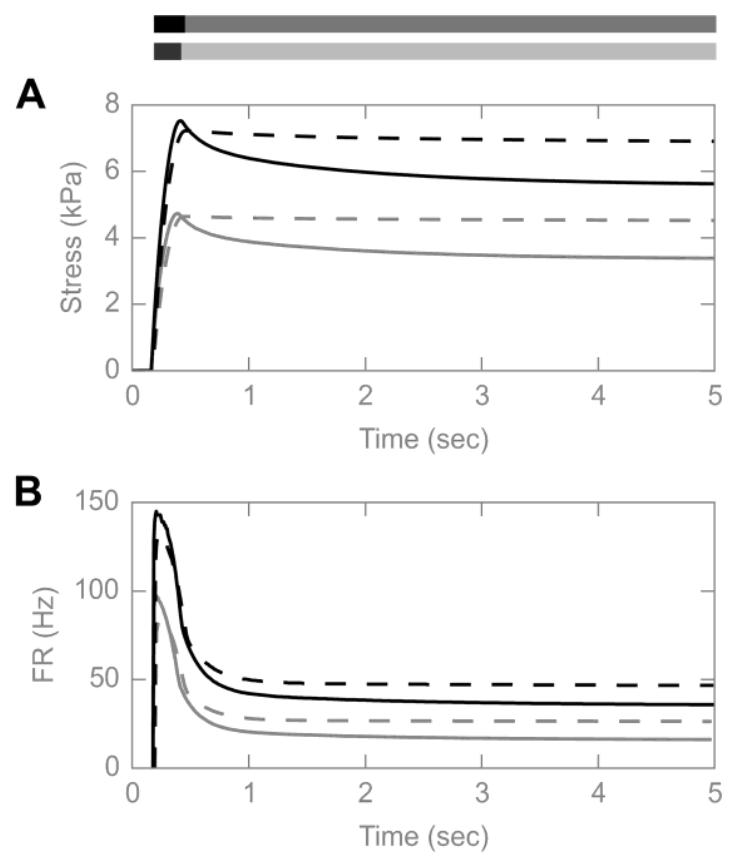

Figure 7. The generator function captures the difference between displacement and force control strategies by producing firing rates with less difference between peak and steadystate values for force-controlled (dashed lines) than displacement-controlled (solid lines and dots) stimulation. (A) Stress over time for displacement- and force-controlled stimulations, with similar peak values generate (B) firing rates. 

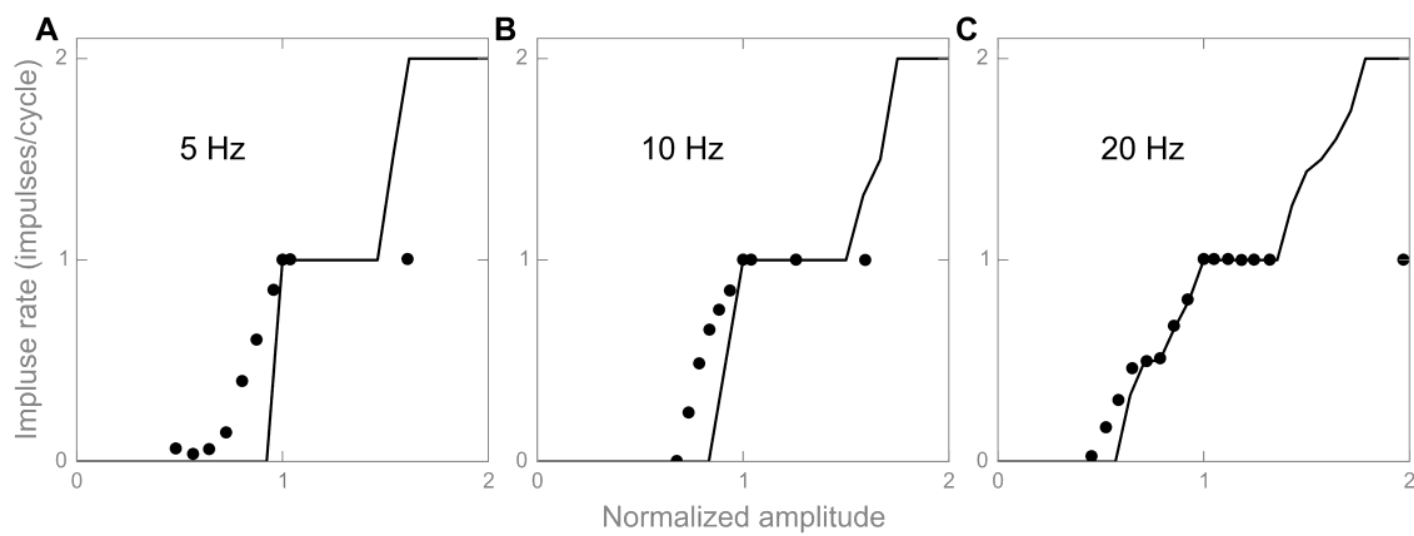

Figure 8. Rate-intensity functions predicted by the model (solid lines) from 5, 10, and 20 $\mathrm{Hz}$ are compared with neural recordings (circles) (Freeman \& Johnson, 1982a). Note that this result is a first approximation as the model is built around the skin and end organ structure of the mouse while the neural recordings are in the primate. Also, no attempt has been made to tune the free parameters for the vibrational case. 

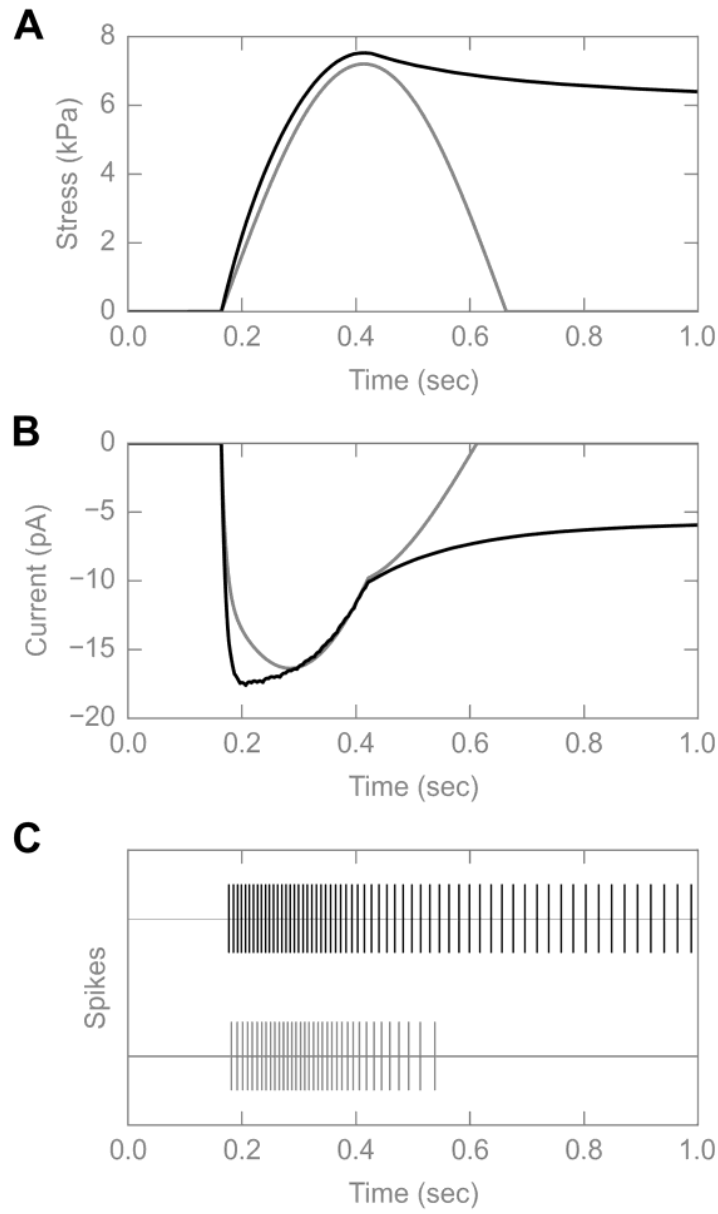

Figure 9. A comparison of outputs from a ramp-and-hold displacement (black lines) and a sinusoidal vibration (grey lines) shows the generator function's capability to produce multiple spikes/cycle for sinusoidal vibration at low frequency. In particular are shown (A) stress over time for a ramp-and-hold displacement and a half sinusoid along with (B) current output over time from the generator function and (C) spike trains. 


\section{Discussion}

To better understand how Merkel cells and neurites respectively contribute to the receptor current that underlies the elicitation of trains of action potentials, this computational effort constructed a generator function that includes both Merkel cell (slower decay in current) and neurite (faster decay in current) components, tied to studies of Piezo2 deficient channels in mice. By convoluting these components with internal stress of the skin, the generator function is able to carry prior receptor currents forward in time, without storing a discrete history of values at prior timestamps. Focusing on biophysical means of transformation, its manner of computation differs from prior efforts that directly converted time derivatives of stimulus position into current. Overall, the model shows how a slowly adapting spike firing response to a sustained hold stimulus, characteristic of the SAI afferent, can emerge from the combination of two receptor currents on different timescales.

The generator function is tied to biophysical measurements with few free parameters. The generator function is modeled to the level of the biology question under study, at the Merkel cell and neurite mechanisms. A model with lower level biology details may be developed in the future, such as on the order of a Hodgkin-Huxley model, once the measurements are possible to attain for containing parameters. 
The model reflects recent findings for Piezo2 deficient mice. In alignment with studies on Piezo2 (Woo et al., 2014) as well as Atoh1 deficient mice (Maksimovic et al., 2014), the muting of the model's Merkel cell mechanism attenuates the firing rate in the stimulus hold. In so doing, only the neurite mechanism's response remains, as observed during stimulus movement and the early hold of the stimulus ( $\sim 0.5 \mathrm{sec}$ after peak force) when the skin is still relaxing to the displacement-controlled stimulus, held at a position in space. This stated, there are several model assumptions that are necessary to arrive at this result. One assumption is that the overall receptor current is simply the superposition of two current sources, at a 16: 1 ratio (neurite: Merkel cell). A second assumption is that the current level and decay of the Merkel cell mechanism is linearly related to the membrane potential recorded for Merkel cells under current-clamped preps. Herein, we relied upon measurements from a current-clamped prep, because the transmission from the Merkel cell to the neurite is likely synaptic in nature. A third assumption is that the current level and decay of the neurite is similar to that measured in the neuron. Furthermore, there are certainly other considerations. For example, our level of abstraction does not account for how the current at the Merkel cell is transmitted to the neurite, which is likely via either a synapse-like transmission between a Merkel cell and a neurite or cell to cell communication. This is as of yet an ongoing debate. A fourth assumption is that we account only for single Merkel cell to single neurite interaction, yet Merkel cells can connect in both chains and 
clusters (Ebara, Kumamoto, Baumann, \& Halata, 2008) and during skin renewal Merkel cells and neurites come and go such that an end organ may have some neurites absent Merkel cells for a period of time.

Similar to Johnson's stratification (Freeman \& Johnson, 1982b), we propose that in modeling the SAI afferent, at least three input-output factors are included: 1) surface stimuli propagates towards its internal layers (skin mechanics), 2) local tissue deformation is converted into current at neurites (generator function), and 3) receptor current is converted into potential and the generation of spikes (neural dynamics). Per this definition, this work is focused upon the generator function. In contrast, other efforts have used the term "transduction" to describe the relationship between receptor current and spike firing, or between surface stimulus and spike firing (Kim et al., 2010). In comparison to those efforts, the model herein integrates time decay constants into the stress to current interaction, rather than the current to voltage interaction. Also, those decay constants were not tied to particular biopotential measurements. Furthermore, we herein integrates into it the notion of carrying forward the recent past history of current.

The Merkel cell and neurite mechanisms of the generator function, together, are convoluted with the internal stress in the skin and this affords a means of storing the prior history of the stimulus, via the present value of receptor current, in a decaying fashion. There might be other computational means of storing the history. For example, the history 
stored at three most recent timestamps in the past, and summed up at present. However such a means would require three points of storage and one point of calculation, and not be naturalistic for a neuron. The chosen means does not require unnatural neuron memory at multiple past timestamps, because the carry-forward characteristic. Therefore, this function demonstrates a natural way of generating and preserving the receptor current temporally.

The generator function can be applied to both ramp-and-hold and vibratory stimuli. Previously, for example, ramp-and-hold stimuli have been delivered under both displacement-control (Lesniak et al., 2014; Sripati et al., 2006) as well as force-control (Wang, Marshall, Baba, Lumpkin, \& Gerling, 2013). In contrast, other studies have modeled periodic vibratory stimuli in the SAI and other afferent types (Freeman \& Johnson, 1982b; Kim et al., 2010). Dividing modeling strategies by such temporal variance in the stimulus has largely led to independent efforts. Herein the focus was upon ramp-and-hold stimuli, though base cases with sinusoidal vibrations are demonstrated for 5,10 , and $20 \mathrm{~Hz}$ data to which the SAI afferent well responds. Prior efforts (Freeman \& Johnson, 1982a; Kim et al., 2010; Slavik \& Bell, 1995) fit the neural recording data much better. This is in part because our model is built for the mouse while the neural data center around the primate, and as well the models in those prior efforts being directly fitted to that data, something not done herein. While we produce here a reasonable first approximation and demonstration of capability, the neural dynamics sub-model (instantiated as a leaky 
integrate-and-fire) would need to be replaced with a more apt alternative if one was to seriously consider and account for millisecond spike timing prediction, phase retardation, and saturation.

The generator function utilizes only one free parameter, which ultimately may be constrainable to a biopotential measurement. In specific, there are three fixed parameters $\left(\tau_{1}, \tau_{2}, K_{1}\right.$ and $K_{2}$, where $\left.K_{1}+K_{2}=1\right)$, and two free parameters $(a$ and $b)$ that are fitted. However, latter two parameters are common by a ratio, leaving only one free parameter. Those parameters $a$ and $b$, which represent the linear transformation from instantaneous stress to instantaneous current of neurite and Merkel cell mechanisms, respectively, are not presently measurable. Although the absolute values of them are unknown, their ratio of 16:1 indicates that a significantly larger portion of effect on the receptor current comes from neurite-generated mechanism, ensures more current (and thereby spike firing) in the stimulus movement phase as opposed to the later sustained hold, and ties to afferents being at least 10 times more sensitive to movement (Johnson, Yoshioka, \& Vega-Bermudez, 2000). In addition, increasing the magnitudes of parameters $\tau_{l}, \tau_{2}$, and $K_{l}$ (decaying time constants and peak/steady ratio) independently increase receptor currents in ramp-up, early-hold, and late-hold phases, respectively, as noted in Figure 4. The ratio of these parameters can also change the ratio of the firing rates in different phases of the stimulus. For example, decreasing $\tau_{l}$ will decrease the overall ramp-up firing rate magnitudes only, 
and therefore can result a decrease of ramp-up : late-hold firing rate ratio. Decreasing $a: b$ from 16:1 to, say, 10:1, will decrease the firing rate ratio of ramp-up:late-hold as well. Finally, the ratio of $a: b$, though at present not constrainable by biopotnetial measurement, could be potentially in the future by either spike or current recordings at the neuron by comparing Piezo2 deficient and wildtype mice.

The generator function explains the neurite mechanism with a rapid-adapting decay, mainly in the ramp-up phase of a stimulation, and the Merkel cell mechanism with a slowly-adapting decay, mainly in the hold phase of a stimulation. It begins to mimic the spike firing features for both normal and Piezo2 deficient mice. That said, further work is needed to mimic the early-hold phase of the stimulus, and perhaps changes could be made to introduce an intermediate-adapting decay, as has been reported in DRG neurons (Ranade et al., 2014). To fully account for the intermediate-adapting response, there might be two approaches. In a first, one might modify both time constants, different from the recordings to which we fit in this work, in the generator function, so that the neurite mechanism response shows both rapid- and intermediate-adapting features as observed in recordings from Piezo2 deficient mice. Another approach might be to add a third exponential term in the neurite mechanism to focus on mimicking intermediate-adapting responses. Such an approach might lend credence to a hypothesis to find a yet to be discovered biological mechanism. 


\section{Methods}

\subsection{Conceptual Understanding of the Generator Function}

The conceptual mechanics of the generator function are demonstrated by magnifying the view of compressive stress internal to the skin (Figure 10A), generated by a finite element model of skin mechanics in response to a ramp-and-hold stimulus, to show the impact of small, discretized step stresses $\left(\sigma_{1}, \sigma_{2}-\sigma_{1}\right.$, etc.) in creating receptor current. In reality, stress output by the skin mechanics model is continuous but a discrete representation demonstrates the following concepts more readily. In Figure 10B, top, the generator function representing a single Merkel cell-neurite complex is input with one instantaneous step stress with value $\sigma_{l}$ at time $t_{l}$, where $\sigma_{l}$ is a very small. In response, in Figure 10B, middle-top, the generator function produces an instantaneous current response $\left(I_{l}\right)$ linear to the stress value $\sigma_{l}$ at time $t_{l}$. Its value decreases over time to a stimulus held at that level of stress. $I_{l}$ is composed of a fast-decaying current $I_{N R}$ (Figure 10B, bottom) from the neurite mechanism, and a slow-decaying current $I_{M C}$ (Figure 10B, middle-bottom) from the Merkel cell mechanism. In Figure 10C, increasing stress over time and the generated current response is demonstrated. A second step stress at time $t_{2}$ is added, making the total stress $\sigma_{2}$ at time $t_{2}$. In response, the current response increases to $I_{2}$ from the $I_{1}$ value which formed at $\sigma_{l}$ and then began to decay over time. A third step stress at time $t_{3}$ 
is then added, making the total stress $\sigma_{3}$ at time $t_{2}$. In response, the current response increases to $I_{3}$ and will decay back to the baseline if there is no further stress input. In Figure 10D, we mimic the case of a decay in stress beginning at peak force. Assuming the stress decays from $\sigma_{n}$ to $\sigma_{n+1}$ at time $t_{n+1}$, the current response drops immediately to $I_{n+1}$, which is of a magnitude linearly related to the absolute change in stress, before continuing to decay in the fashion described in Figure 10B.

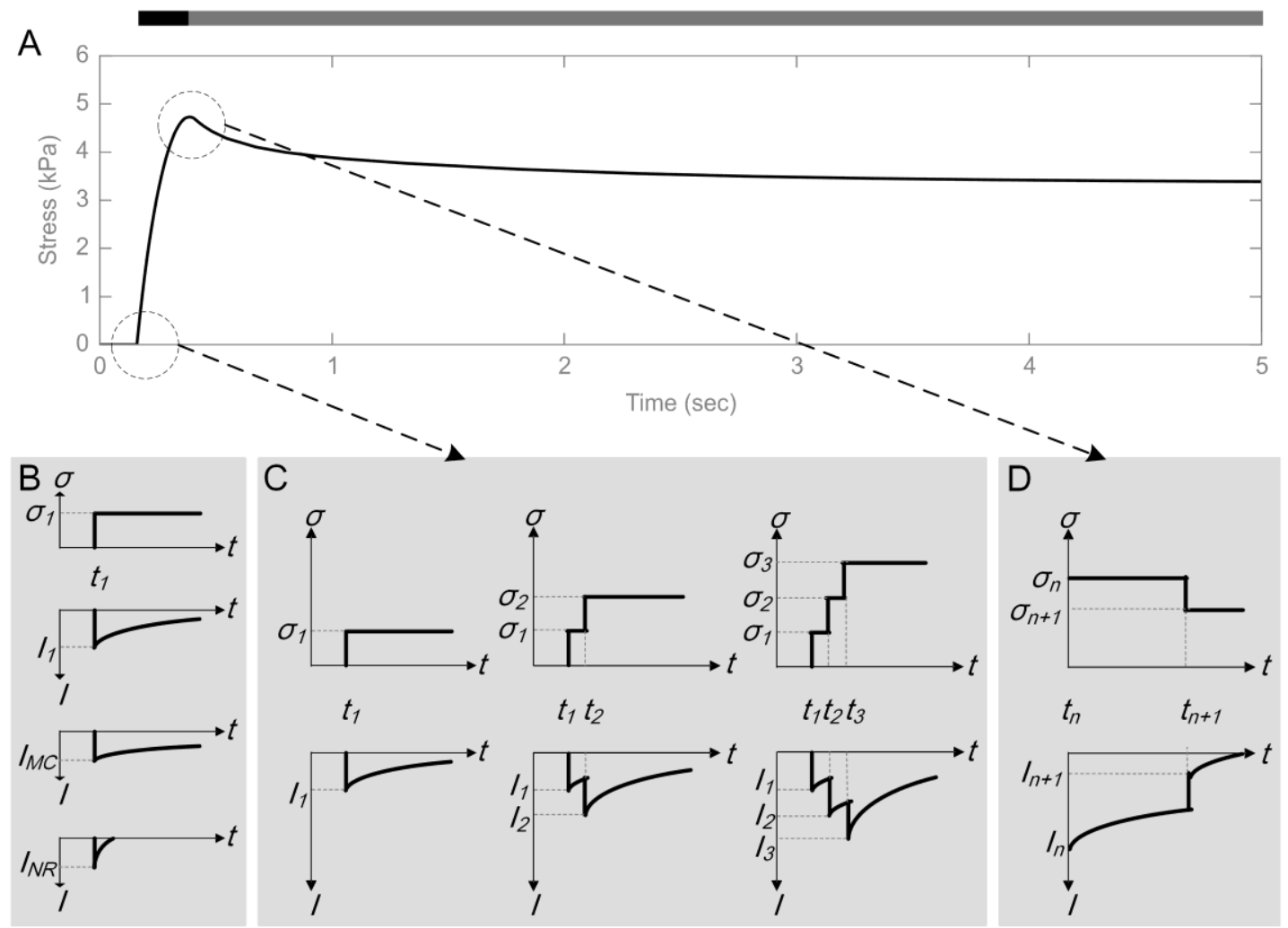

Figure 10. In a conceptual view of the generator function, (A) stress over time, under a displacement-controlled ramp-and-hold stimulation, serves as the input. Then, in three cases with inputs of step stresses, (B) a single step increase in stress $\sigma_{l}$ evokes current 
output $I_{l}$, which is the sum of Merkel cell $I_{M C}$ and neurite $I_{N R}$ mechanisms, (C) three sequentially delivered step stresses show that current decays but builds upon the prior magnitude, and (D) a single step decrease in stress from $\sigma_{n}$ to $\sigma_{n+1}$ evokes an immediate decrease in current followed by a slower decay.

\subsection{Mathematical Form of the Generator Function}

The generator function is a convolution of internal compressive stress in the simulated skin and two exponential functions that describe how a single Merkel cell-neurite complex responds to a step stimulation input with an instantaneous increase or decrease proportional to stress magnitude followed by exponential decay. To better mimic the current generated from a Merkel cell and a neurite, we use two exponential functions, representing either respectively. Electrophysiological recordings suggest that current in a neuron rapidly decays with a step stimulation, and we assume that a neurite behaves similarly. Therefore, the neurite part of Eqn. 1 corresponds to decay time constant $\tau_{l}$ and linear transformation coefficient $a$. The Merkel cell part of Eqn. 2 corresponds to decay time constant $\tau_{2}$, linear transformation coefficient $b$, and two ratio parameters $K_{l}$ and $K_{2}$, representing the peak and steady portions of a decaying trace (where $K_{l}+K_{2}=1$ ). Note that linear transformation coefficients $a$ and $b$ serve to convert instantaneous stress to instantaneous current, linearly, 
and are set at a ratio of $16: 1$, respectively, such that the neurite is more sensitive than the Merkel cell. This and many of the model assumptions are addressed in section 4 . Discussion.

$$
\begin{aligned}
& a * \exp \left(-\frac{t-x}{\tau_{1}}\right) \\
& \quad b *\left(K_{1} * \exp \left(-\frac{t-x}{\tau_{2}}\right)+K_{2}\right)
\end{aligned}
$$

Bringing Eqns. 1 and 2 into the bracket of Eqn. 3, the complete form of the generator function is a convolution of these terms and the first derivative of stress input $\sigma$ over time (Eqn. 3):

$$
I(t)=\int_{x=0}^{t}\left[a * \exp \left(-\frac{t-x}{\tau_{1}}\right)+b *\left(K_{1} * \exp \left(-\frac{t-x}{\tau_{2}}\right)+K_{2}\right)\right] * \frac{d \sigma}{d x} d x
$$

where $I$ is the output generator current, $t$ is time, and $x$ is a variable of the integral. We use 0 as the mathematical baseline of $I$, and set it to 0 when it becomes negative. The terms $a$ and $b$ are instantaneous values while $\frac{d \sigma}{d x}$ along with the integral represents their decay over time, which is the means of storing the prior history of the stimulus, via the present value of receptor current, in a decaying fashion.

\subsection{Generator Function in context of SAI Whole End Organ Model}

Since we cannot directly measure the receptor currents in a neurite that would emerge from the contribution of Merkel cell and neurite mechanisms, the generator function was validated in the context of an end-organ model for the SAI afferent (Lesniak et al., 2014) 
(Figure 11). In this model, one Merkel cell and its connecting neurite form a Merkel cellneurite complex, where multiple complexes are clustered per heminode. For example, the end-organ structure in Figure 11 shows 4 heminodes and therefore 4 clusters, with 3,1,5, and 8 Merkel cell-neurite complexes in each, noted as a $\{8,5,3,1\}$ structure (sequence does not matter). In each Merkel cell-neurite complex, a finite element model of the skin's layers outputs compressive stress internal to the skin given a stimulus input of displacement with a linear decelerating ramp-up. Different from prior work (Lesniak et al., 2014), a refined finite element was used that was both hyper and viscoelastic as based directly upon measurements of the mouse, and using the output of maximum compressive stress instead of strain energy density. Each layer of the multi-layered model was represented by axisymmetric hybrid elements of quasi-linear viscoelastic material with Ogden and NeoHookean hyperelastic components (Wang, Baba, Lumpkin, \& Gerling, n.d.). In response to indentation by ramp-and-hold and periodic vibration stimuli, its output of compressive stress over time is passed to the generator function, which calculates receptor current for one Merkel cell-neurite complex. Then, receptor current is multiplied by the number of Merkel cell-neurite complexes in a cluster as the total current entering the heminode, which is taken in a leaky integrate-and-fire model to accumulate enough potential to elicit a spike. There is therefore one LIF model at each heminode. Once the potential at a heminode reaches the firing threshold and elicits a spike, the potentials at other heminodes are 
immediately reset to baseline, and a refractory period of $1 \mathrm{msec}$ is set. The parameters R, C, and V (resistance, capacitance, and firing voltage threshold) of the LIF model are set to 3E-11 F, 16.67E8 Ohm, and $30 \mathrm{mV}$, and are the same for all 4 LIF models in the model.

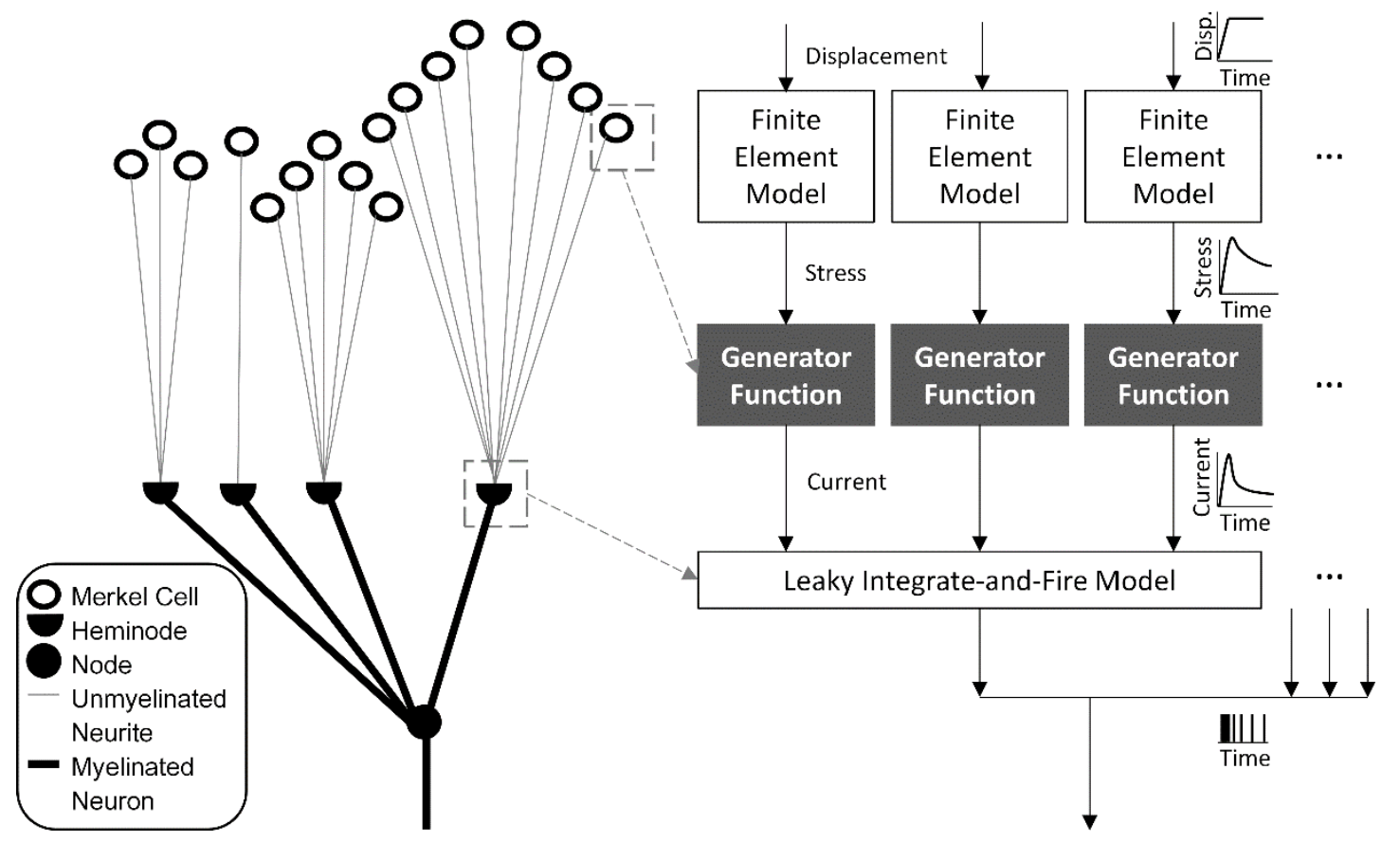

Figure 11. A model of the end-organ of an SAI afferent (Lesniak et al., 2014) used in validating the generator function. The model takes stimulation input on the skin to a finite element model and outputs stress in the skin near the locations of the Merkel cells (hollow circles) and unmyelinated neurites (grey lines). Stress then enters the generator function to form receptor currents. The currents in each cluster of Merkel cell-neurite complexes are summed and input to leaky integrate-and-fire models at the sites of heminodes (half circles) to fire spikes, which propagate through myelinated neurons 
(black lines) to an integrated node (circle) where spike trains can be measured.

\subsection{Fitting the Parameters of the Generator Function}

The free parameters of the generator function are $a$ and $b$ while its biologically-derived parameters are $\tau_{1}, \tau_{2}, K_{1}$, and $K_{2}$. While the latter parameters were obtained experimentally, the former parameters needed to be fitted and could only be fitted in the context of simulating an entire end organ.

Regarding the biologically derived parameters, time constant $\tau_{1}$ in the neurite mechanism was fitted to the decay time constant obtained from the current recorded in the neuron of a whole cell over time under a voltage clamped prep, with step mechanical stimulation (Ranade et al., 2014). We assume similarity of current decay between such neurons and the simulated neurites used herein. A characteristic recording and its fitted trace are shown in Figure 12B. As shown in Table 2, a total of 44 measurements from nine preps were fitted using a single exponential decay functions of the form $y=a * \exp \left(-\frac{x}{\tau}\right)$. The mean value of all fitted time constants, $0.008 \mathrm{sec}$, was used for $\tau_{1}$, and the mean value \pm standard deviation of all fitted time constants, 0.005 and $0.013 \mathrm{sec}$, were used in numerical experiments with parameter changes. In contrast, the time constant $\tau_{2}$, as well as the peak to steady state ratio parameters $K_{l}$ and $K_{2}$ of the Merkel cell mechanism were 
generated directly from single isolated Merkel cells. In this case, however, membrane potential over time was recorded in the current clamped prep (Woo et al., 2014). We assume that the Merkel cell's transmission mechanism most likely behaves like a synapse where changes in the cell membrane's potential are linearly related to post-synaptic current under a step stimulation. A characteristic recording and its fitted trace shown in Figure 12A. A total of 12 voltage measurements from three Merkel cells were fitted using a similar single exponential decay plus a constant function. The mean value of all fitted time constants (Table 3), $0.2 \mathrm{sec}$, was used for $\tau_{2}$, and the mean value \pm standard deviation of all fitted time constants, 0.05 and $0.35 \mathrm{sec}$, were used in numerical experiments with parameter changes. The mean value of all fitted ratios $\left(\frac{a}{a+b}\right.$ and $\left.\frac{b}{a+b}\right)$ (Table 4), 0.5 and 0.5 , was used for $K_{l}$ and $K_{2}$, where $K_{l}+K_{2}=1$, and the mean value \pm standard deviation of all fitted $K_{l}$ 's, 0.1 and 0.9 , were used in numerical experiments with parameter changes $\left(K_{2}\right.$ changes to 0.9 and 0.1 , respectively). 

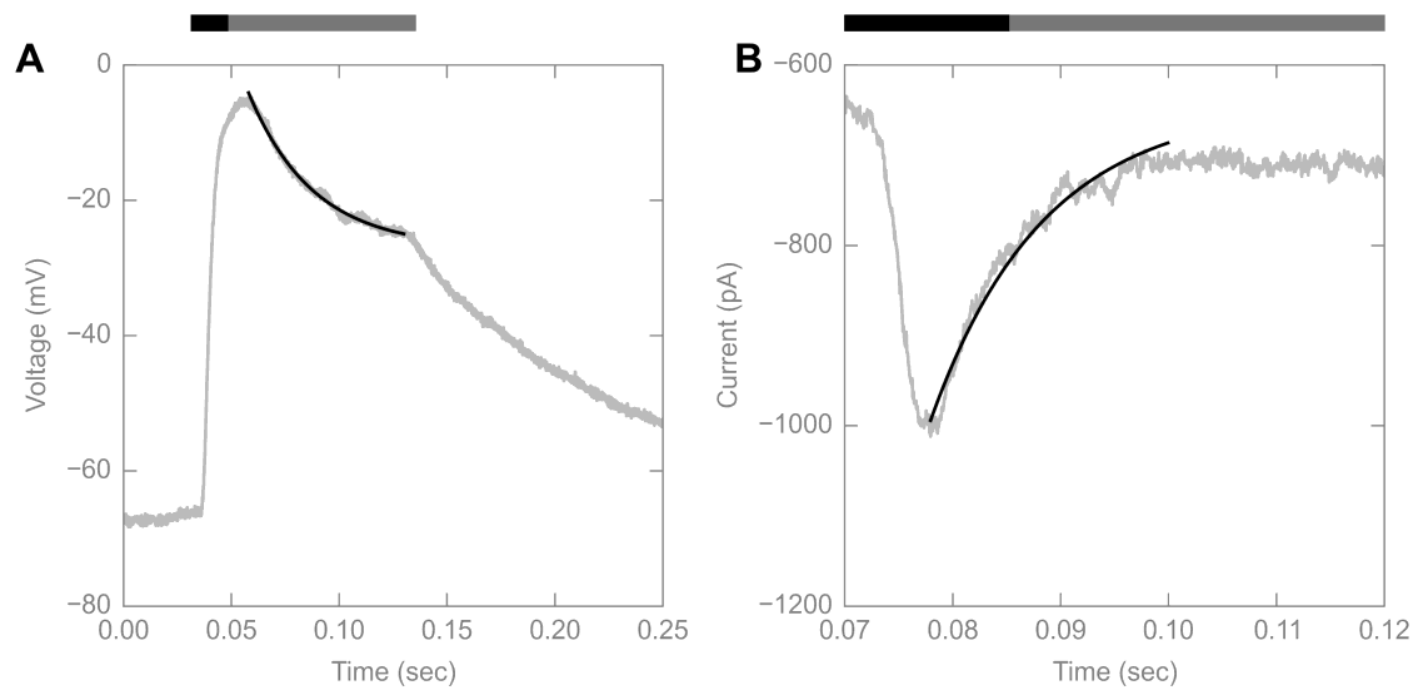

Figure 12. The parameters $\tau_{1}$ and $\tau_{2}$ of generator function are fitted from the recording data, where (A) shows a characteristic trace of Merkel cell membrane potential over time under a current clamped prep, delivered a step mechanical stimulus at about $88 \%$ of the saturation threshold, and (B) shows a characteristic trace of current recorded in the neuron of a whole cell over time under a voltage clamped prep, delivered a mechanical stimulus of about $85 \%$ of the saturation threshold. Note that the bar at top shows a darken section corresponding to stimulus movement into the skin and the lighter section showing the stimulus hold.

Table 2. Time constants in milliseconds for fitted traces of current recordings in the SAI afferent in response to a step mechanical stimulation near the end organ (fitting $\tau_{1}$ : mean = $8 \mathrm{msec}$, stdev $=5 \mathrm{msec}$ ). 


\begin{tabular}{|l|c|l|l|l|l|l|l|l|l|}
\hline & Run 1 & Run 2 & Run 3 & Run 4 & Run 5 & Run 6 & Run 7 & Run 8 & Run 9 \\
\hline Fiber 1 & 8.2 & & & & & & & & \\
\hline Fiber 2 & 5.4 & 5.6 & 10.4 & 11.4 & 8.2 & 8.9 & & & \\
\hline Fiber 3 & 31.3 & 15.8 & 14.8 & & & & & & \\
\hline Fiber 4 & 9.6 & 11.0 & 17.1 & & & & & & \\
\hline Fiber 5 & 7.5 & 4.3 & 2.7 & 2.8 & 3.1 & 3.9 & 3.2 & & \\
\hline Fiber 6 & 4.8 & 4.5 & 7.3 & 5.4 & 9.5 & 5.8 & & & \\
\hline Fiber 7 & 4.7 & 2.6 & 5.2 & 2.7 & 3.7 & 6.4 & 4.0 & 5.6 & 4.3 \\
\hline Fiber 8 & 3.4 & & & & & & & & \\
\hline Fiber 9 & 5.9 & 4.4 & 6.9 & 15.0 & 9.9 & 6.1 & 7.9 & 14.5 & \\
\hline
\end{tabular}

Table 3. Time constants in milliseconds for fitted traces of potential recordings for isolated Merkel cells in response to a step mechanical stimulation (fitting $\tau_{2}$ : mean $=200 \mathrm{msec}$, stdev $=150$ msec $).$

\begin{tabular}{|l|c|c|c|c|c|}
\hline & Run 1 & Run 2 & Run 3 & Run 4 & Run 5 \\
\hline Fiber 1 & 163.7 & 555.6 & 288.2 & 387.6 & \\
\hline Fiber 2 & 42.8 & 50.3 & 342.5 & 30.0 & 43.0 \\
\hline Fiber 3 & 7.2 & 4.0 & 7.9 & & \\
\hline
\end{tabular}


Table 4. Ratio of potential in Merkel cell recordings from the peak value to the steady state value in response to a step mechanical stimulation (fitting $K_{l}$ ).

\begin{tabular}{|l|c|c|c|c|c|}
\hline & Run 1 & Run 2 & Run 3 & Run 4 & Run 5 \\
\hline Fiber 1 & 0.41 & 1.00 & 0.88 & 1.00 & \\
\hline Fiber 2 & 0.19 & 0.33 & 1.00 & 0.36 & 0.50 \\
\hline Fiber 3 & 0.21 & 0.26 & 0.27 & & \\
\hline
\end{tabular}

Regarding the free parameters $a$ and $b$ that required fitting by the end organ model, the magnitude of one mechanism relative to another, as well as the way in which the Merkel cell current is transferred to the neurite and mixes with it are unknown. As typical values of currents in whole afferent recordings can reach up to $250 \mathrm{pA}$ (Lumpkin, personal communication), and that our model contains 17 Merkel cell-neurite complexes to achieve this value, we estimated the receptor currents from a single Merkel cell-neurite complex should be evenly divided by 17 , with a peak value of $10-20$ pA. With this as a starting point, their values were fitted in the whole end-organ model so that firing rates predicted over the ramp-up, early-hold, and late-hold phases of the stimulus mimic the electrophysiological recordings in Table 1, for an afferent described elsewhere (Wang et al., n.d.). The final values used for $a$ and $\mathrm{b}$ are $3 \mathrm{E}-14 \mathrm{~A} / \mathrm{Pa}$ and $1.875 \mathrm{E}-15 \mathrm{~A} / \mathrm{Pa}$, respectively, with a ratio of $16: 1$. 


\subsection{Numerical Experiments with the Generator Function in Context of the End Organ Model}

We explored how the variance of each time constant decay $\tau_{1}, \tau_{2}$ and the peak to steady state ratio $K_{1}$, and $K_{2}$ would affect the generator current and the corresponding firing rate. As described in Section 5.4, the standard deviations of the parameters were set in the generator function. The values of $\tau_{l}$ were varied between 5,8 , and $13 \mathrm{msec}$. The values of $\tau_{2}$ were varied between 50,200, and $350 \mathrm{msec}$. The values of $K_{l}$ were varied between 0.1, 0.5 , and 0.9 . The firing rates calculated from the spike trains, as well as the ratio of firing rates at different phases of the stimulus (ramp-up, early-hold and late-hold) were compared to electrophysiological recordings under similar stimulation.

The next sets of numerical experiments were all run with the same set of parameters described in Section 5.3 and 5.4. The parameters a, b, $\tau_{1}, \tau_{2}, K_{1}$, and $K_{2}$ are set to be $3 \mathrm{E}-14$ $\mathrm{A} / \mathrm{Pa}, 1.875 \mathrm{E}-15 \mathrm{~A} / \mathrm{Pa}, 8 \mathrm{msec}, 200 \mathrm{msec}$, 0.5, and 0.5. The parameters $\mathrm{R}, \mathrm{C}$, and $\mathrm{V}$ (resistance, capacitance, and firing voltage threshold) of the LIF model are set to 3E-11 F, 16.67E8 $\mathrm{Ohm}$, and $30 \mathrm{mV}$, and are the same for all 4 LIF models in the model.

To mimic the phenomenon that mice deficient in Piezo2 channels in the skin do not generate a firing rate response under sustained stimulation, we muted the Merkel cell mechanism part of the generator function. We compared the shape of the model predictions 
to electrophysiological recordings with normal and Piezo2 deficient mice.

To validate the generator function under different types of stimulation, we ran the model under ramp-and-hold stimulations in both displacement- and force-control, as well as periodic vibration. To compare the model predictions for displacement- and forcecontrolled stimulations, we used stimuli that generated similar magnitudes of peak stress in the skin, and compared the ratio of firing rates across the phases of stimulation. To compare periodic vibration at different frequencies, we used half sinusoids of generated stress as input and varied peak magnitudes to fire zero to two spikes per cycle. The impulse rate is the average number of spikes per cycle over $5 \mathrm{sec}$ of stimulation. The stimulation amplitude is normalized by the amplitude at the first point of 1 impulse per cycle for every trace. Then, to compare sinusoidal vibration to ramp-and-hold stimulations, we used half sinusoids of stresses with $1 \mathrm{~Hz}$ frequency, so that the duration of the ramps were similar. 


\section{References}

Dong, Y., Mihalas, S., Kim, S. S., Yoshioka, T., Bensmaia, S., \& Niebur, E. (2013). A simple model of mechanotransduction in primate glabrous skin. Journal of Neurophysiology, 109(5), 1350-9. doi:10.1152/jn.00395.2012

Ebara, S., Kumamoto, K., Baumann, K. I., \& Halata, Z. (2008). Three-dimensional analyses of touch domes in the hairy skin of the cat paw reveal morphological substrates for complex sensory processing. Neuroscience Research, 61(2), 159-171. doi:10.1016/j.neures.2008.02.004

Freeman, A. W., \& Johnson, K. O. (1982a). A model accounting for effects of vibratory amplitude on responses of cutaneous mechanoreceptors in macaque monkey. $J$. Physiol., 323(1), 43-64. Retrieved from http://jp.physoc.org/content/323/1/43. short

Freeman, A. W., \& Johnson, K. O. (1982b). Cutaneous mechanoreceptors in macaque monkey: temporal discharge patterns evoked by vibration, and a receptor model. $J$. Physiol., 323(1), 21-41. Retrieved from http://jp.physoc.org/content/323/1/21. short

Johnson, K. O., Yoshioka, T., \& Vega-Bermudez, F. (2000). Tactile functions of mechanoreceptive afferents innervating the hand. Journal of Clinical 
Neurophysiology: Official Publication of the American Electroencephalographic

Society, 17(6), 539-558. doi:10.1097/00004691-200011000-00002

Kim, S. S., Sripati, A. P., \& Bensmaia, S. J. (2010). Predicting the timing of spikes evoked by tactile stimulation of the hand. Journal of Neurophysiology, 104(3), 1484-96. doi:10.1152/jn.00187.2010

Lesniak, D. R., \& Gerling, G. J. (2009). Predicting SA-I mechanoreceptor spike times with a skin-neuron model. Mathematical Biosciences, 220(1), 15-23. doi:10.1016/j.mbs.2009.03.007

Lesniak, D. R., Marshall, K. L., Wellnitz, S. A., Jenkins, B. A., Baba, Y., Rasband, M. N., .. Lumpkin, E. A. (2014). Computation identifies structural features that govern neuronal firing properties in slowly adapting touch receptors. eLife, 3, e01488. doi:10.7554/eLife.01488

Looft, F. J., \& Baltensperger, C. M. (1990). Linear Systems Analysis of Cutaneous Type I Mechanoreceptors. IEEE Transactions on Biomedical Engineering, 37(6).

Maksimovic, S., Nakatani, M., Baba, Y., Nelson, A. M., Marshall, K. L., Wellnitz, S. A., ... Lumpkin, E. A. (2014). Epidermal Merkel cells are mechanosensory cells that 
tune mammalian touch receptors. Nature, 509(7502), 617-21.

doi:10.1038/nature13250

Ranade, S. S., Woo, S.-H., Dubin, A. E., Moshourab, R. a, Wetzel, C., Petrus, M., ... Patapoutian, A. (2014). Piezo2 is the major transducer of mechanical forces for touch sensation in mice. Nature, 516(7529), 121-5. doi:10.1038/nature13980

Slavik, P., \& Bell, J. (1995). A mechanoreceptor model for rapidly and slowly adapting afferents subjected to periodic vibratory stimuli. MathematicalBiosciences, 130(1), 1-23. doi:10.1016/0025-5564(94)00083-C

Sripati, A. P., Bensmaia, S. J., \& Johnson, K. O. (2006). A continuum mechanical model of mechanoreceptive afferent responses to indented spatial patterns. Journal of Neurophysiology, 95(6), 3852-64. doi:10.1152/jn.01240.2005

Wang, Y., Baba, Y., Lumpkin, E. A., \& Gerling, G. J. (n.d.). Surface pressure as the native tactile cue is robustly encoded by the skin amidst changes in its properties. Journal of Neurophysiology.

Wang, Y., Marshall, K. L., Baba, Y., Lumpkin, E. A., \& Gerling, G. J. (2013). Natural variation in skin thickness argues for mechanical stimulus control by force instead of 
displacement. In 2013 World Haptics Conference, WHC 2013 (pp. 645-650). Daejeon, Korea: IEEE. doi:10.1109/WHC.2013.6548484

Wellnitz, S. A., Lesniak, D. R., Gerling, G. J., \& Lumpkin, E. A. (2010). The regularity of sustained firing reveals two populations of slowly adapting touch receptors in mouse hairy skin. Journal of Neurophysiology, 103(6), 3378-88. doi:10.1152/jn.00810.2009

Woo, S.-H., Lumpkin, E. a, \& Patapoutian, A. (2015). Merkel cells and neurons keep in touch. Trends in Cell Biology, 25(2), 74-81. doi:10.1016/j.tcb.2014.10.003

Woo, S.-H., Ranade, S., Weyer, A. D., Dubin, A. E., Baba, Y., Qiu, Z., ... Patapoutian, A. (2014). Piezo2 is required for Merkel-cell mechanotransduction. Nature, 509(7502), 622-6. doi:10.1038/nature13251 